\title{
Experimental Study on Relationship Between Critical State Line and Zero Creep Volume Change Zone in Engineered Silty Sand
}

\author{
Young-Hoon Jung ${ }^{1, *}$, Taesun Lee ${ }^{1}$, Ka-Hyun Park $^{2}$, and Choong-Ki Chung ${ }^{3}$ \\ ${ }^{1}$ Kyung Hee University, Dept. of Civil Engineering, 1732 Deogyeong-daero, Yongin-si, Republic of Korea \\ ${ }^{2}$ Korea Institute of Construction and Building Technology, Republic of Korea \\ ${ }^{3}$ Seoul National University, Dept. of Civil and Environmental Engineering, Republic of Korea
}

\begin{abstract}
Volume change during creep is one of the important factors to explain various time-dependent problems such as the pile set-up effect. Park [1] and Park et al. [2] experimentally measured creep volume characteristics under various stress conditions using engineered silty sand obtained from weathered granite soil in Korea. They found that a zero-creep volume change zone (ZCVZ) exists on the e-log p' plane. If the void ratios at the beginning of the creep are above the ZCVZ on the e-log p' plane, the volume decreases during creep; if they are below the ZCVZ, the volume increases during creep. In this study, a series of simple critical state (CS) tests which can simply determine the critical state parameters in sandy soils were performed to estimate the critical state line (CSL) and compared with ZCVZ. In addition, the CSL obtained using the method proposed by Santamarina and Cho [3] was compared with those obtained by the triaxial test. The CSL for Jumunjin sand and engineered silty sand is close to ZCVZ. Therefore, the CSL can be used as a reference line to determine the volume change pattern by creep.
\end{abstract}

\section{Introduction}

The problem of long-term displacement of structures built on granular soil has been frequently reported. For example, the setup phenomenon, in which the shaft capacity increases by several times its initial value in the years following installation of the driven piles in granular soils, is a typical problem related to the time-dependent behaviour of granular soil. Among several sources causing such long-term engineering problems in granular soils, the creep is highly likely an influential factor.

Creep refers to deformation of a material over time under a constant load. To model the deformation behaviour of creep, information on volumetric strain and shear strain during creep is crucial. Unfortunately, most of previous studies deals only with changes in axial or shear strain during creep, and studies dealing with volumetric deformation behaviours during creep are very limited.

Murayama et al. [4] reported volume dilation during creep at high stress ratios in a triaxial compression test on Toyoura sand. Mejia et al. [5] showed via a triaxial test that the stress ratio, confining pressure, grain angularity, and density could affect the magnitude of shear and volumetric strains during creep. Lade and Liu [6] showed the variations in axial and volumetric strains during creep after applying various confining pressures and stress ratio conditions to dense sand. Kuwano and Jardine [7] reported the variations in volumetric and shear strains during creep after applying various stress ratio conditions to loose and dense sands. Bowman and Soga [8] reported an increase in volume over time during creep in dense sand at a relatively high stress ratio. Lade et al. [9] measured volumetric strain in creep tests under various stress conditions using crushed coral sand, and they reported that the initial volumetric deformation during creep reflected the volumetric deformation that occurred during the previous loading stage. Rimoy and Jardine [10] reported that volume deformation during creep was contractive at first and then dilative later, using tests with loose or medium-dense sands. Karimpour and Lade [11] reported a dilative volume change when a high stress ratio was applied to dense sand under a low confining pressure; this type of change was related to the volumetric deformation just before creep.

The results of the aforementioned studies suggested that, while the volumetric creep strain of granular soil under low stress ratios was contractive, dense granular soil under high stress ratios exhibited dilative behaviours, although such behaviour was greatly dependent on density [8]. Therefore, the main factors that determine the volumetric creep deformation behaviour of granular soil were identified as soil density and creep stress state; however, it is unclear which combinations of conditions for these two factors result in contractive or dilative volume deformation of granular soil during creep.

In this study, experimental results for the volumetric creep strain behaviour of a non-plastic silty sand under various void ratios and creep stress conditions are presented. It was found that the volumetric deformation behaviour of silty sand during creep, which was either contractive or dilative, is determined by a specific

\footnotetext{
* Corresponding author: jyounghoon $@$ khu.ac.kr
} 
combination of void ratio $\left(e_{c}\right)$ and mean normal effective stress $\left(p_{c}^{\prime}\right)$ at the beginning of creep. From the experimental results, the combinations of $e_{c}$ and $p_{c}^{\prime}$ that do not cause volume change during creep were determined, and the zero-creep volume change zone (ZCVZ) was defined. Contraction or dilation in the volumetric creep strain pattern can be predicted according to the conditions of $e_{c}$ and $p_{c}^{\prime}$ for the ZCVZ on an e-log $\mathrm{p}^{\prime}$ plane. The mechanism of the observed volumetric creep strain behaviour of engineered silty sand is explained through the dislocation slip and recovery process in the creep of metal.

In addition, the CSL obtained using the method proposed by Santamarina and Cho [3] and the CSL for similar materials reported in the existing literature were compared with those obtained by the triaxial test. The band of CSL can be found by combining the results obtained by various methods. The CSL for the engineered silty sand is quite similar to ZCVZ. Therefore, CSL can be used as a reference line to determine the volume change pattern by creep.

\section{State-dependent volume change during creep}

Park [1] and Park et al. [2] experimentally measured creep volume characteristics under various stress conditions using engineered silty sand obtained from weathered granite soil in Korea. Soil collected from a construction site near Cheonggye Mountain in Seoul, South Korea, was used as the raw material for this study. The soil used in this study was a non-plastic silty sand with 5\% clay (smaller than $0.02 \mathrm{~mm}), 26 \%$ silt $(0.02-0.075 \mathrm{~mm})$, and $44 \%$ fine sand (between $0.075 \mathrm{~mm}$ and $0.425 \mathrm{~mm}$ ). The specific gravity of the tested soil was 2.67. The maximum and minimum void ratios of the test soil were 0.59 and 1.61 , respectively. X-ray fluorescence analysis showed that the major minerals of the sampled soil were quartz and muscovite. According to the Unified Soil Classification System (USCS), the test material is classified as a silty sand (SM).

For conducting the creep tests in the triaxial testing equipment, nine stress conditions using different combinations of the initial void ratio, $e_{0}$, the mean normal effective stress, $p^{\prime}=\left(\sigma_{a}^{\prime}+2 \sigma_{r}^{\prime}\right) / 3$, and the deviator stress, $q=\sigma_{a}^{\prime}-\sigma_{r}^{\prime}$, where $\sigma_{a}^{\prime}$ and $\sigma_{r}^{\prime}$ represent the axial and radial effective stresses were selected. Three different values of $e_{0}$ of $0.63,0.75$, and 0.87 were prepared.

Table 1. Stress conditions for creep tests.

\begin{tabular}{|c|c|c|c|c|}
\hline Test ID & $\begin{array}{c}p^{\prime} \\
(\mathrm{kPa})\end{array}$ & $\begin{array}{c}q \\
(\mathrm{kPa})\end{array}$ & $\begin{array}{c}\sigma_{a}^{\prime} \\
(\mathrm{kPa})\end{array}$ & $\begin{array}{c}\text { Stress ratio, } \\
\eta=q / p^{\prime}\end{array}$ \\
\hline 1 & 100 & 0 & 100 & 0.0 \\
2 & 82.24 & 17.65 & 100 & 0.2 \\
3 & 78.95 & 31.58 & 100 & 0.4 \\
4 & 200 & 0 & 200 & 0.0 \\
5 & 176.47 & 35.29 & 200 & 0.2 \\
6 & 157.89 & 63.16 & 200 & 0.4 \\
7 & 400 & 0 & 400 & 0.0 \\
8 & 352.94 & 79.59 & 400 & 0.2 \\
9 & 315.79 & 126.32 & 400 & 0.4 \\
\hline
\end{tabular}

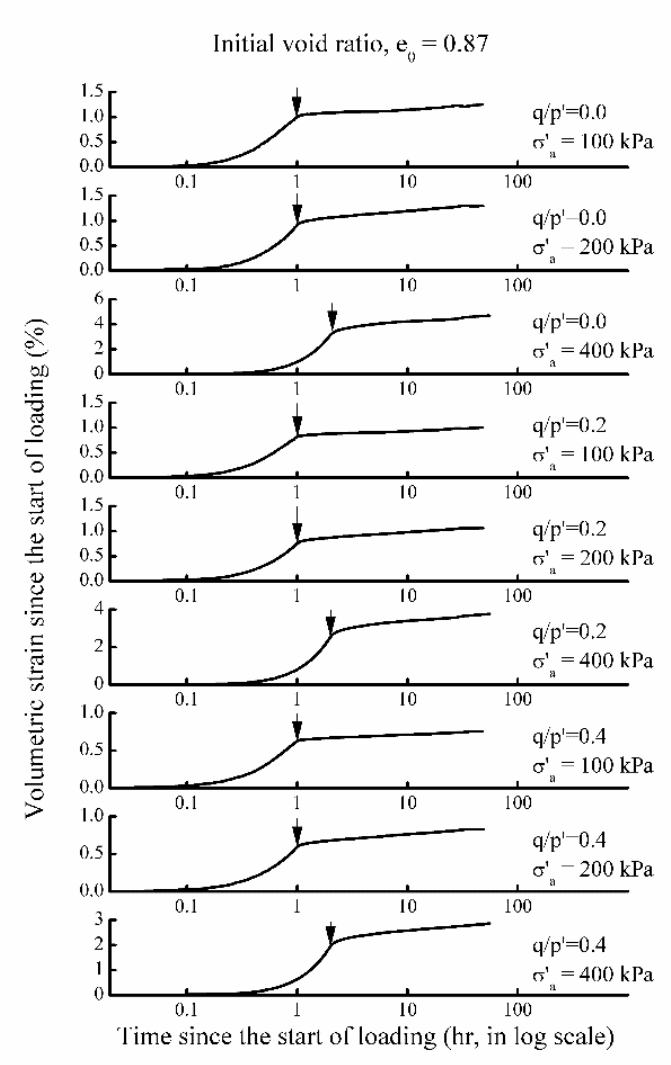

Fig. 1. Variation of volumetric strains since the start of loading and during consolidation and creep for $e_{0}=0.87$ [2].

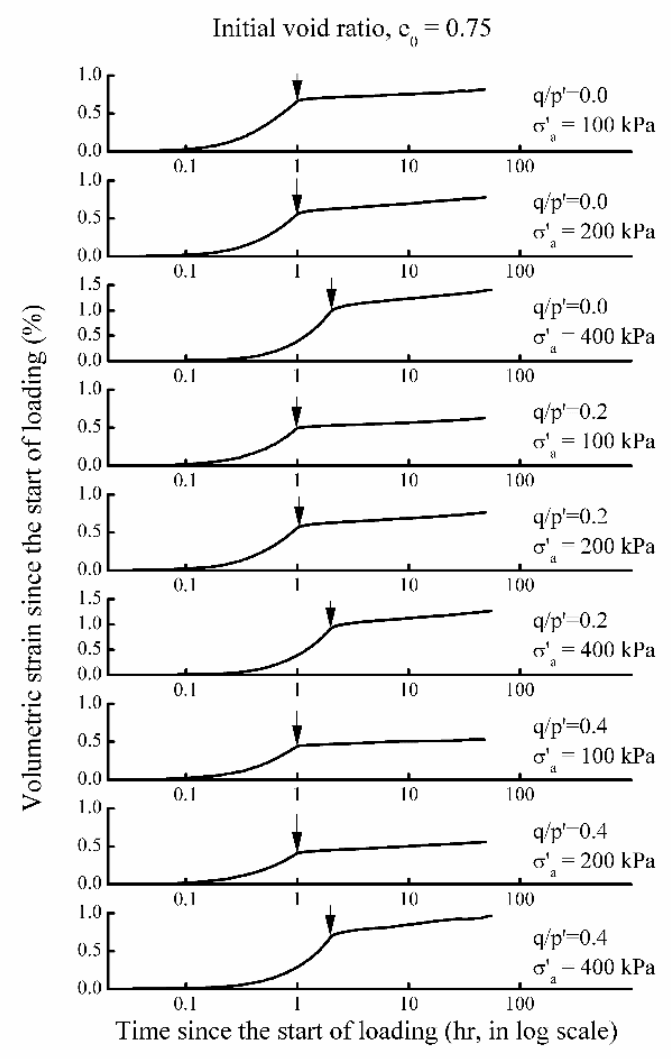

Fig. 2. Variation of volumetric strains since the start of loading and during consolidation and creep for $e_{0}=0.75$ [2]. 


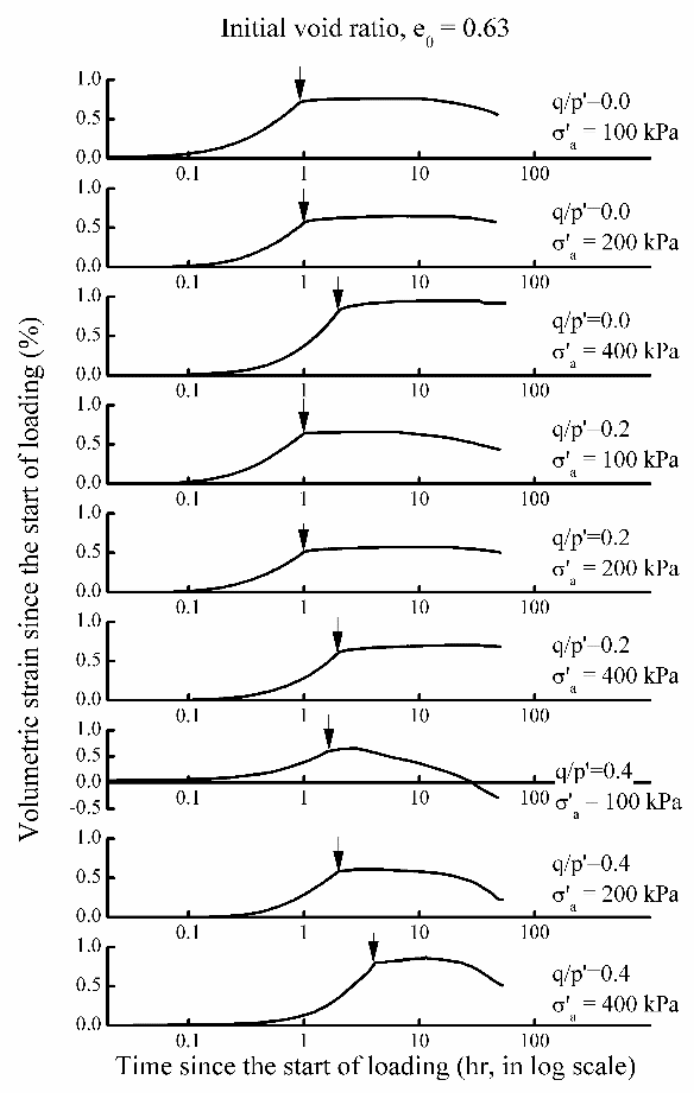

Fig. 3. Variation of volumetric strains since the start of loading and during consolidation and creep for $e_{0}=0.63$ [2].

Fig. 1, 2, and 3 show the variation of volumetric strains since the start of loading and during consolidation and creep. The small arrows in figures mark the start time of the creep test. As shown in figures, the higher the stress ratio, the lower the volumetric creep strain developed. As shown in Fig. 3, under the low confinement of $\sigma_{a}^{\prime}=100$ $\mathrm{kPa}$ and $\eta=0.0$ the volumetric creep strain, $\epsilon_{v o l}^{c r}$, decreased in a dilative manner after $15 \mathrm{~h}$ of the creep test time, $t_{\text {creep }}$. Under the higher stress ratio of $0.4, \epsilon_{v o l}^{c r}$ become more dilative during creep.

Fig. 4 shows the changes in void ratio from $e_{0}$ to the void ratio at the beginning of the creep test, $e_{c}$, and subsequent value of the void ratio at $t_{\text {creep }}=40 \mathrm{~h}, e_{40}$. The change of void ratio from $e_{0}$ to $e_{c}$ was drawn by a vector of which the length was increased by a factor of three. The steady creep state void ratio, $e_{s c s}$, was defined as the void ratio at which the creep strain rate was no longer changing after sufficient elapse of $t_{\text {creep }}$. For analysis of the data of volumetric creep strains, it is assumed that the volumetric creep strain of the soil, either dilating or contracting, will eventually cease to change and, thus, will reach a steady state. Unlike shear deformation, the volume or density of soil is likely to have a certain limit to be changed under a given stress condition during creep.

As shown in Fig. 4, a single straight line regressing the points of $e_{s c s}$ for the same $e_{0}$ in the $e-\log p^{\prime}$ plot. This line is defined as the steady creep state line (SCSL). There are three SCSL corresponding to three different $e_{0}, 0.67$, 0.75 , and 0.87 , as marked by blue lines in Fig. 4. Along with these SCSLs, a shaded band indicates the zero-creep volume zone $(\mathrm{ZCVZ})$ that covers the scatter of the data points of the zero-creep volume change void ratio, $e_{z c v}$.

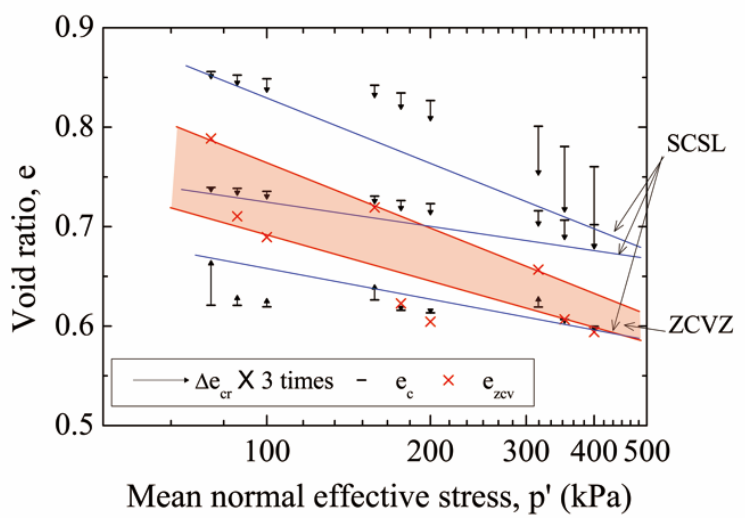

Fig. 4. Variation of void ratio during creep, steady creep state line (SCSL), and zero-creep volume change zone (ZCVC) [2].

In the void ratio of a sample at the beginning of the creep test is equal to $e_{z c v}$, there were no change or a minor change in volume during creep. A single $e_{z c v}$ exists for a given $p^{\prime}$ at a specific value of $q / p^{\prime}$.

Fig. 4 explains how the volume of soil and its void ratio change under specific conditions of $e_{c}$ and $p_{c}^{\prime}$ during creep. If $e_{c}$ and $p_{c}^{\prime}$ fell within the $\mathrm{ZCVZ}$ at the beginning of creep, the volume change during creep was very small. If $e_{c}$ and $p_{c}^{\prime}$ fell above the ZCVZ, the volume of soil and its void ratio continued to decrease until they reached the SCSL, but they did not reach the ZCVZ. If $e_{c}$ and $p_{c}^{\prime}$ fell below the $\mathrm{ZCVZ}$ on the $e-\log p^{\prime}$ plane, the volume of soil and its void ratio increased until they reached the SCSL, but, again, they did not reach the ZCVZ. Therefore, the behavior of volume change during creep was determined by the positions of $e_{c}$ and $p_{c}^{\prime}$ relative to the $\mathrm{ZCVZ}$ on the $e-\log p^{\prime}$ plane, and the changes in the void ratio during creep proceeded toward the ZCVZ. However, the change of the void ratio toward $\mathrm{ZCVZ}$ ceases at the SCSL of a given $e_{0}$ possibly due to a limitation of the external energy supplied by the constant load during creep. Three blue lines marked as SCSL are an example of this assumption.

These characteristics were very similar to the pattern of volume change in soil that depends on the position of the void ratio and the confining stress relative to the critical-state line on the $e-\log p^{\prime}$ plane, according to the critical-state theory. However, unlike the critical-state theory, wherein the void ratio at the steady state during shearing is the same as the void ratio at the critical state, the void ratio for the steady creep state did not reach the $\mathrm{ZCVZ}$ but the SCSL corresponding to the given $e_{0}$ in creep test.

\section{Simple critical state tests}

Previously, the concept and its existence of the SCSL and ZCVZ were described. It is naturally of interest that SCSL and $\mathrm{ZCVZ}$ are compared with the critical state line (CSL) in the $e-\log p^{\prime}$ plane. To measure the CSL, we adopted 
the method of the simple critical state (CS) test proposed by Santamarina and Cho [3]. Two different soils, Jumunjin standard sand and engineered silty sand that was the same material used for the creep tests described previously, were used for the simple CS tests. Table 1 summarizes material properties.

The simple CS test requires testing equipment including vacuum, thin latex membrane, two plexiglass caps, two O-rings, a graduated cylinder, a porous stone, and a transparent hose. Once the dimensions of the components such as the internal cross-sectional area of the tube and the volume of the device components were measured, the unit weight of water, $\gamma_{w}$, and the specific gravity, $G_{s}$, needed to be determined. The critical state friction angle, $\phi_{c s}$, can be measured by pouring soil in the graduated cylinder filled with water. As shown in Fig. 5, the angle of repose in the middle region of the slope was measured as $\phi_{c s}$. The measured values of $\phi_{c s}$ for Jumunjin sand and Engineered silty sand are 24.9 and 32.3 degrees, respectively.

Table 1. Material properties of tested soils.

\begin{tabular}{|c|c|c|}
\hline Material & Jumunjin sand & $\begin{array}{c}\text { Engineered silty } \\
\text { sand }\end{array}$ \\
\hline Specific gravity, $G_{s}$ & 2.62 & 2.67 \\
\hline$C_{u}$ & 1.24 & 9.02 \\
\hline$C_{c}$ & 0.96 & 0.80 \\
\hline$D_{50}(\mathrm{~mm})$ & 0.52 & 0.18 \\
\hline$D_{10}(\mathrm{~mm})$ & 0.43 & 0.028 \\
\hline$e_{\max }$ & 0.91 & 1.52 \\
\hline$e_{\min }$ & 0.61 & 0.69 \\
\hline$\phi_{c s}$ & $24.9^{\circ}$ & $32.3^{\circ}$ \\
\hline
\end{tabular}

The critical state void ratios for the different confining stress were measured separately. To prepare the specimen, the soil was gradually spooned into the membrane filled with water until the soil occupies a predetermined height about twice the diameter of the latex membrane. A top plexiglass cap was placed by displacing the excess water. Prior to testing, maximum vacuum was applied and then the specimen was forcedly kneaded to remove any possible entrapped air. After sufficient saturation of the specimen was ensured, the vacuum was released. The specimen was subjected to a low vacuum pressure, $\sigma_{c}^{\prime}$, and subsequently loaded by hand until axial strain approached approximately $40 \%$. The elevation of the water caused by loading was recorded. This procedure was repeated using different $\sigma_{c}^{\prime}$ of $20,40,60$ and $85 \mathrm{kPa}$. Fig. 7 shows the photos taken at each stage of the simple CS test.

The values of $p^{\prime}$ and $q$ at the critical state can be evaluated by

$$
p^{\prime}=\sigma_{c}^{\prime} \frac{3-\sin \phi_{c s}}{3\left(1-\sin \phi_{c s}\right)} \text { and } q=\sigma_{c}^{\prime} \frac{2 \sin \phi_{c s}}{1-\sin \phi_{c s}}
$$

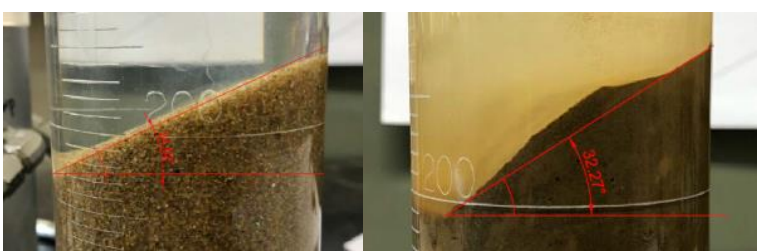

Fig. 5. Measurement of the critical state friction angle, $\phi_{c S}^{\prime}$, of (a) Jumunjin sand and (b) Engineered silty sand

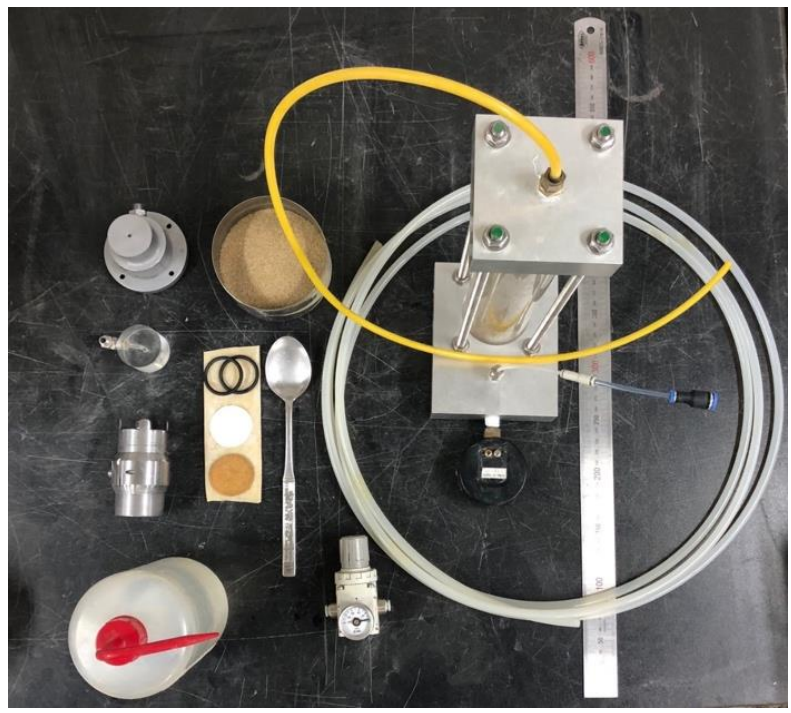

Fig. 6. Testing equipment for the simple CS test.

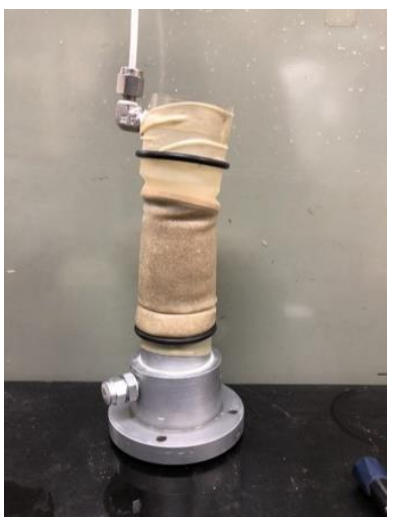

(a)

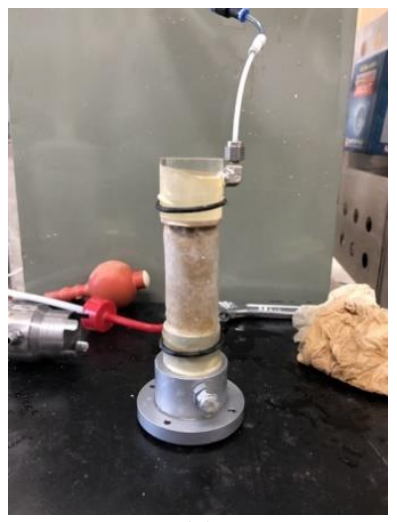

(c)

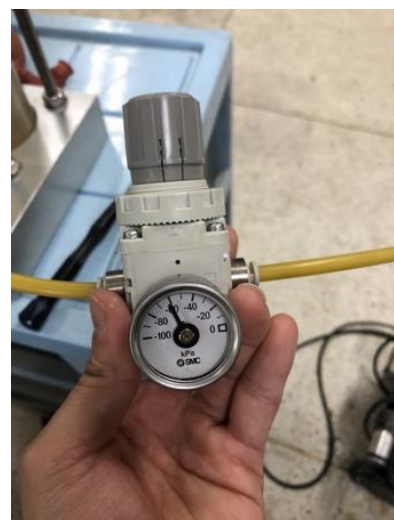

(b)

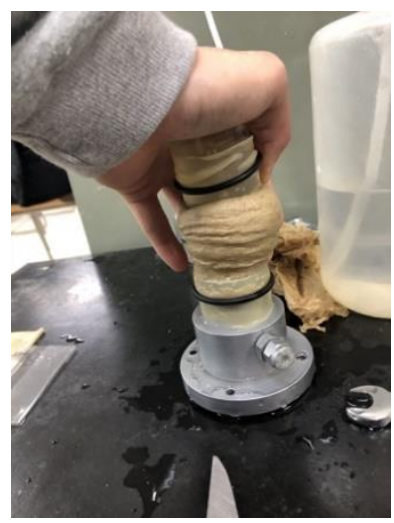

(d)
Fig. 7. Test procedure: (a) after sample preparation, (b) applying the vacuum at a given magnitude, (c) the standing sample with vacuum, (d) the specimen sheared by hand. 
The critical state void ratio at a given $p^{\prime}$ can be calculated from the initial volume and the measured volume changes in the transparent hose.

\section{Results and discussions}

Figure 8 compares the measured critical state void ratios at $p^{\prime}=35.3,70.6,105.8$, and $149.9 \mathrm{kPa}$ corresponding to the vacuum pressures of $20,40,60$ and $85 \mathrm{kPa}$ for engineered silty sand and at $p^{\prime}=29.7,44.6,59.4$, and $89.1 \mathrm{kPa}$ corresponding to the vacuum pressures of 20,30 , 40 and $60 \mathrm{kPa}$ for Jumunjin sand. The shear test was repeated five times at a given $p^{\prime}$ for engineered silty sand. As shown in Fig. 8, a best fitted line over the data points can be drawn and taken as the critical state line (CSL) in the $e-\log p^{\prime}$ plane. For comparison, other reported CSLs of ASTM graded sand, sandboil sand, Ottawa 2030 sand, and glass beads [3] are plotted together in Fig. 8. As can be found in Fig. 8, the slope of the CSL in the $e-$ $\log p^{\prime}$ plane is 0.184 and 0.254 for the engineered silty sand and Jumunjin sand, respectively. These values are comparatively higher than the slopes for other soils presented in Fig. 8 ranging between 0.04 and 0.08 .

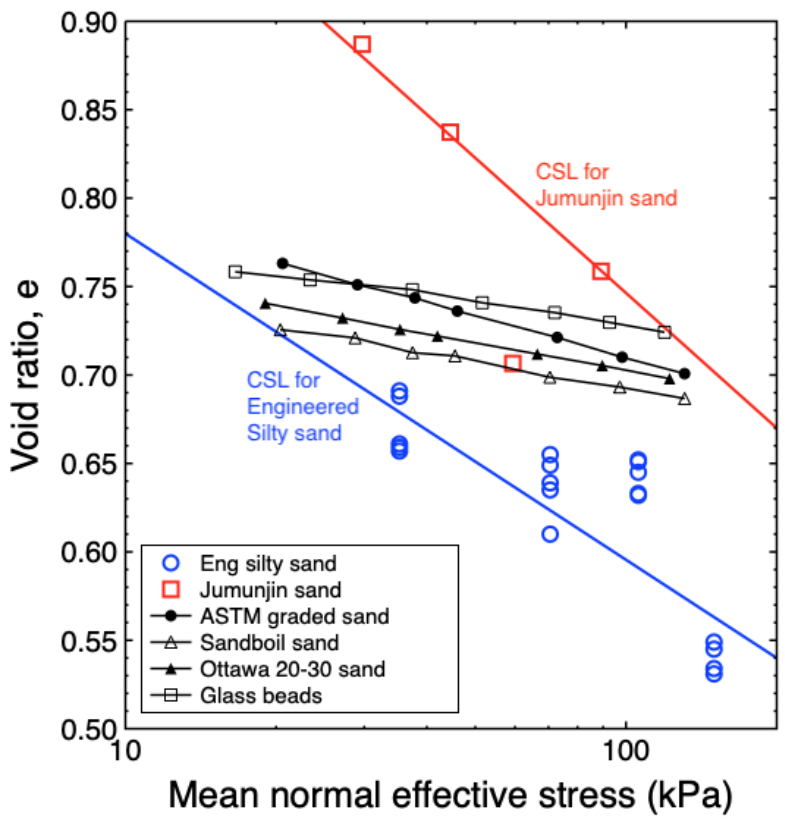

Fig. 8. Critical state lines measured by the simple CS tests.

Figure 9 compares the CSL of the tested soil with the slope of the ZCVZ shown in Fig. 4. The slope of the ZCVZ in the $e-\log p^{\prime}$ plane ranges between 0.15 for the lower boundary line and 0.22 for the upper boundary line. Therefore, the slope of the CSL of the engineered silty sand is close to those of the ZCVZ. In Fig. 9, the upper and lower boundary lines of the ZCVZ are drawn by linearly extending the ZCVZ boundaries presented in Fig. 4. Although the slopes of the CSL and ZCVZ are similar, however, the position of the CSL quite deviates from those of the $\mathrm{ZCVZ}$ in the $e-\log p^{\prime}$ plane. At the same range of the mean normal effective stress, the difference of the void ratio between the CSL and the ZCVZ is approximately 0.2 at the maximum. Differences in the testing device configuration and the size of the specimen used for measuring CSL and ZCVZ may attribute such deviation in the void ratio. The possible errors in the measurement of the initial void ratio for the engineered silty sand could be a likely source of this deviation. It is of interest that the slope and position of the CSL for Jumunjin sand is quite similar to those of the ZCVZ for engineered silty sand.

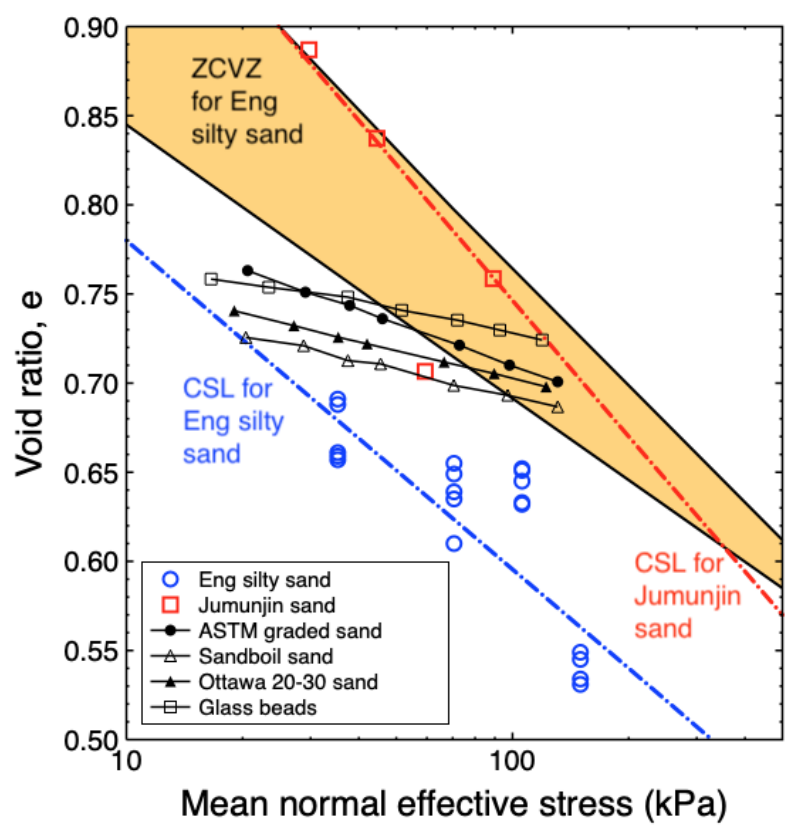

Fig. 9. Comparison between the CS lines with ZCVZ.

\section{Conclusions}

In this study, a series of the simple CS test was conducted for two different sandy soils. The critical state lines (CSL) in the $e-\log p^{\prime}$ plane from these tests were compared with the CSL for other material as well as the ZCVZ for the engineered silty sand. From the test results, the following interesting conclusions were drawn.

(1) The volume contraction or dilation behaviours during creep are determined by the void ratio and the mean normal effective stress at the beginning of creep.

(2) A ZCVZ exists on the $e-\log p^{\prime}$ plane. If $e_{c}$ and $p_{c}^{\prime}$ are above the ZCVZ on the plane, the volume decreases during creep; if they are below the ZCVZ, the volume increases during creep.

(3) With respect to the slope in the $e-\log p^{\prime}$ plane, the CSL measured by the simple CS tests is comparable to the ZCVZ for the engineered silty sand. The slope and position of the CSL for Jumunjin sand is close to those of the ZCVZ for the engineered silty sand. The $\mathrm{CSL}$ is a good alternative to the ZCVZ as a reference to determine the volume change pattern by creep.

This work was supported by the National Research Foundation of Korea (NRF) grant funded by the Korea government (MEST) (No. NRF-2015R1A2A2A01005969) and 'NST*-KICT $\dagger$ Postdoctoral Research Fellowship for Young Scientists' at Korea 
Institute of Civil Engineering and Building Technology in South Korea.

* National Research Council of Science \& Technology / $\uparrow$ Korea Institute of Civil Engineering and Building Technology

\section{References}

1. K.-H. Park, Ph.D thesis, Seoul National University (2016)

2. K.-H., Park, C.-K. Chung, Y.-H. Jung, J. Geotech. Geoenviron. (to be published)

3. J. C. Santamarina, G. C. Cho, Geotech. Test. J. 24, 185 (2001)

4. S. Murayama, K. Michihiro, T. Sakagami, Soils Found. 24, 1-15 (1984)

5. C. Mejia, Y. P. Vaid, D. Negussey, Proc., Int. Conf. on Rheology and Soil Mech., Coventry, UK, 312-326 (1988)

6. $\quad$ P. V. Lade, C.-T. Liu, J. Eng. Mech. 124, 912 (1988)

7. R. Kuwano, R. J. Jardine, Can. Geotech. J. 39, 1061 (2002)

8. E. T. Bowman, K. Soga, Soils Found. 43, 107-117. (2003)

9. P. V. Lade, C. D. Liggio Jr, J. Nam, J. Geotech. Geoenviron. Eng. 135, 941 (2009)

10. S. P. Rimoy, R. J. Jardine, R. J. Proc., 5th Int. Symp. on Deformation Characteristics of Geomaterials, ISSeoul 2011, IOS Press, 463 (2011)

11. H. Karimpour, P. V. Lade, Can. Geotech. J. 50, 1159 (2013). 